\title{
BMJ Open Self-testing for pregnancy: a systematic review and meta-analysis
}

To cite: Kennedy CE, Yeh PT, Gholbzouri K, et al. Selftesting for pregnancy: a systematic review and meta-analysis. BMJ Open 2022;12:e054120. doi:10.1136/ bmjopen-2021-054120

- Prepublication history and additional supplemental material for this paper are available online. To view these files, please visit the journal online (http://dx.doi.org/10.1136/ bmjopen-2021-054120).

Received 03 June 2021 Accepted 01 February 2022

Check for updates

(C) Author(s) (or their employer(s)) 2022. Re-use permitted under CC BY-NC. No commercial re-use. See rights and permissions. Published by BMJ.

${ }^{1}$ Department of International Health, Johns Hopkins University Bloomberg School of Public Health, Baltimore, Maryland, USA

${ }^{2}$ World Health Organisation Regional Office for the Eastern Mediterranean, Cairo, Egypt ${ }^{3}$ Department of Sexual and Reproductive Health and Research, World Health Organization, Geneva, Switzerland

Correspondence to Dr Manjulaa Narasimhan; narasimhanm@who.int

\author{
Caitlin E Kennedy, ${ }^{1}$ Ping Teresa Yeh (1) , ${ }^{1}$ Karima Gholbzouri, ${ }^{2}$ \\ Manjulaa Narasimhan ${ }^{3}$
}

\begin{abstract}
Objectives Urine pregnancy tests are often inaccessible in low-income settings. Expanded provision of home pregnancy testing could support self-care options for sexual and reproductive health and rights. We conducted a systematic review of pregnancy self-testing effectiveness, values and preferences and cost.

Design Systematic review and meta-analysis using the Grading of Recommendation, Assessment, Development, and Evaluation (GRADE) approach.
\end{abstract}

Data sources PubMed, CINAHL, LILACS and EMBASE and four trial registries were searched through 2 November 2020.

Eligibility criteria for selecting studies We included trials and observational studies that compared urine selftesting for pregnancy to health worker-led pregnancy testing on effectiveness outcomes; quantitative and qualitative studies describing values and preferences of end users and health workers and costs of pregnancy selftesting.

Data extraction and synthesis Two independent reviewers used standardised methods to search, screen and code included studies. Risk of bias was assessed using the Cochrane Collaboration and Evidence Project tools. Meta-analysis was conducted using random effects models. Findings were summarised in GRADE evidence profiles and synthesised qualitatively.

Results For effectiveness, four randomised trials following 5493 individuals after medical abortion showed no difference or improvements in loss to follow-up with home pregnancy self-testing compared with return clinic visits. One additional trial of community health workers offering home pregnancy tests showed a significant increase in pregnancy knowledge and antenatal counselling among 506 clients. Eighteen diverse values and preferences studies found support for pregnancy self-testing because of quick results, convenience, confidentiality/privacy, cost and accuracy. Most individuals receiving pregnancy selftests for postabortion home management preferred this option. No studies reported cost data.

Conclusion Pregnancy self-testing is acceptable and valued by end users. Effectiveness data come mostly from articles on postabortion care, and cost data are lacking. Greater availability of pregnancy self-tests, including in postabortion care and CHW programs, may lead to improved health outcomes.

PROSPERO registration number CRD42021231656.

\section{INTRODUCTION}

Urine tests for pregnancy measure the presence of human chorionic gonadotropin
Strengths and limitations of this study

This systematic review and meta-analysis used a comprehensive search for articles not only on the effectiveness of pregnancy self-testing (both randomised trials and comparative observational studies) but also on values and preferences of end users and health workers and costs.

- We used the Grading of Recommendation, Assessment, Development and Evaluation approach to evaluate the strength and quality of the evidence.

- Because pregnancy self-testing is commonplace in many settings, it is perhaps unsurprising that not many studies have examined the comparative effectiveness of this intervention on home use versus facility-based access, except in specific circumstances like postabortion care and community health worker programmes.

- Values and preferences data came from diverse populations around the world, suggesting high generalisability, but no comparative cost data on pregnancy self-testing were identified.

(hCG) and are widely used to detect pregnancy in both home and clinical settings. Urine pregnancy tests have evolved substantially since they were first developed 100 years ago. ${ }^{1}$ The current generation of tests has close to $100 \%$ sensitivity and specificity in detecting hCG at concentrations of $25 \mathrm{IU} / \mathrm{mL}$ or more, and, thus, are able to detect pregnancy as early as 1 day after a missed menstrual period, if performed per the manufacturers' instructions. ${ }^{1}$ However, test performance varies based on characteristics of the test, such as what form of hCG is detected ${ }^{23}$ as well as user characteristics. A systematic review published in 1998 identified five studies that evaluated the diagnostic efficiency of home pregnancy tests. ${ }^{4}$ Sensitivity ranged from 0.52 to 1.0 across tests. A gradient of sensitivity was identified by user group. Sensitivity was highest in studies where urine samples obtained by the investigators were tested by volunteers (sensitivity: $0.91,95 \%$ CI 0.84 to 0.96 ) and lower in actual patients who performed the test on their own urine samples (sensitivity 
0.75 (95\% CI 0.64 to 0.85$)$ ). Similarly, the test effectiveness score (discriminatory power, where a score of 1.0 or less indicates poor distinctions between pregnant and non-pregnant individuals and higher scores imply greater effectiveness) was 2.75 (95\% CI 2.3 to 3.2) for studies where subjects were volunteers but $0.82(95 \%$ CI 0.4 to 1.2) for studies with actual patients.

Providing pregnancy tests for home use may have a range of benefits for different populations. In Madagascar, randomised trial data have shown that providing pregnancy tests to community health workers (CHWs) for home distribution can increase both engagement in antenatal care services ${ }^{5}$ and contraceptive services, since a negative pregnancy test is necessary before initiating some contraceptive methods. ${ }^{6}$ Home pregnancy tests have been shown to be an acceptable and feasible option for follow-up among couples undergoing assisted reproduction. ${ }^{7}$ There is also evidence supporting the efficacy, safety and acceptability of urine pregnancy tests to confirm the effectiveness of a medical abortion instead of an ultrasound. ${ }^{89}$ While urine pregnancy self-tests are available over-the-counter in many high-income and middleincome settings, in many low-income settings, they may be financially inaccessible to most people outside of public health services, or unavailable altogether, leading individuals with the sole option of health facility-based blood tests to confirm pregnancy. For example, most countries in the Eastern Mediterranean Region have pregnancy self-testing widely available in private pharmacies, particularly in urban settings, and mainly used by the upper socioeconomic class due cost and knowledge. ${ }^{10}$

Many people in resource-constrained settings are not able to decide if, how many, and when to have children; increased access to self-care interventions, such as pregnancy self-tests, could support their health decisionmaking. More widespread efforts to provide pregnancy self-tests that can be used at home or another preferred location could support increased autonomy of individuals as well as support multiple programmatic settings to advance sexual and reproductive health and rights.

We sought to review the literature on home use of pregnancy self-testing as an additional option to facilitybased testing. We conducted this systematic review in the context of expanding the evidence base of the 2019 WHO's normative guidance on self-care interventions ${ }^{11}$ to include new considerations related to sexual and reproductive health. This review was also conducted in the context of the COVID-19 pandemic, which continues to result in significant disruptions of essential sexual and reproductive health services. The restrictive measures taken to prevent the spread of COVID-19, particularly lockdowns, have resulted in the need for increased availability and accessibility to this self-care intervention.

\section{METHODS}

This review addressed the question: should self-testing for pregnancy be available as an additional option to health facility-based testing? We reviewed the extant literature in three areas relevant to answering this question: effectiveness of the intervention, values and preferences of end users and health workers and cost information. The review followed Preferred Reporting Items for Systematic Reviews and Meta-Analysis guidelines, ${ }^{12}$ and the protocol was published on PROSPERO. As this review was conducted to inform a WHO guideline, we followed the WHO Handbook for Guideline Development ${ }^{13}$ and used the recommended Grading of Recommendation, Assessment, Development and Evaluation (GRADE) process ${ }^{14}$ to summarise findings.

\section{Effectiveness review}

The effectiveness review was designed according to the population-intervention-comparison-outcomes (PICO) format as follows:

Population

Individuals seeking pregnancy testing

Intervention

Urine self-testing for pregnancy.

\section{Comparison}

Health worker-led testing for pregnancy (health facility or community clinic with either urine and/or serum test for pregnancy).

\section{Outcomes}

1. Missed ongoing pregnancy.

2. Appropriate clinical follow-up (counselling, antenatal care visit(s), contraceptive services, abortion services, etc).

3. Gestational age at pregnancy awareness (knowledge of pregnancy) and at presentation for antenatal care or abortion.

4. Self-efficacy, self-determination, autonomy and empowerment.

5. Mental health and well-being (anxiety, stress, selfharm).

6. Adverse events and social harms (including stigma, discrimination, coercion, violence (including intimatepartner violence, violence from family members or community members, etc) and breaches of confidentiality), and whether these harms were corrected/had redress available.

7. Device-related issues (eg, test failure, problems with manufacturing, packaging, labelling or instructions for use)

All results that were compatible with each outcome domain in each study were extracted.

\section{Inclusion criteria}

To be included in the review, an article had to meet the following criteria:

1. Study design that compared urine self-testing for pregnancy to health worker-led testing for pregnancy (health facility or community services with either urine 
and/or serum test for pregnancy). This included both randomised controlled trials (RCTs), non-RCTs and comparative observational studies (including prospective controlled cohort studies, cross-sectional studies, controlled before-after studies and interrupted time series) that compare individuals who received the intervention to those who did not.

2. Measured one or more of the outcomes listed above.

3. Published in a peer-reviewed journal.

No restrictions were placed based on location of the intervention. No language restrictions were used on the search. Articles in English, French, Spanish and Chinese were coded directly; articles in other languages were translated.

\section{Search strategy}

The following electronic databases were searched through the search date of 2 November 2020: PubMed, CINAHL, LILACS and EMBASE. We searched for ongoing RCTs through clinicaltrials.gov, the WHO ICTRP, PACTR and the Australian New Zealand Clinical Trials Registry. Secondary reference searching was also conducted on all studies included in the review. Furthermore, selected experts in the field were contacted to identify additional articles not identified through other search methods. The full search strategy was developed for PubMed and adapted for entry into all computer databases (online supplemental appendix A). These search terms were used for the main systematic review (PICO question), for the values and preferences review and for the cost review.

\section{Screening Abstracts}

Titles, abstracts, citation information and descriptor terms of citations identified through the search strategy were screened by a member of the senior study staff. Fulltext articles were obtained of all selected abstracts, and two independent reviewers assessed all full-text articles for eligibility to determine final study selection. Differences were resolved through consensus.

\section{Data extraction and management}

Data were extracted independently by two reviewers using standardised data extraction forms. Differences in data extraction were resolved through consensus, and referral to a senior study team member from WHO when necessary.

The following information was gathered from each included study:

- Study identification: author(s), type of citation, year of publication.

- Study description: study objectives, location, population characteristics, type of urine pregnancy test, description of self-test access, study design; sample size, follow-up periods and loss to follow-up.

- Outcomes: analytic approach, outcome measures, comparison groups, effect sizes, CI, significance levels, conclusions, limitation.s
For randomised trials, risk of bias was assessed using the Cochrane Collaboration's tool for assessing risk of bias. ${ }^{15}$ For non-randomised trials but comparative studies, study rigour was assessed using the Evidence Project 8-item checklist for intervention evaluations. ${ }^{16}$

\section{Data analysis}

Data were analysed according to coding categories and outcomes. Where multiple studies reported the same outcome, meta-analysis was conducted using random effects models to combine risk ratios with the programme comprehensive meta-analysis. ${ }^{17}$ Risk ratios were used directly when provided and were calculated from the number of events and total sample size in the intervention and comparison groups when not reported. Heterogeneity was calculated using the $\mathrm{I}^{2}$ statistic.

We planned to stratify all analyses by the following categories/subgroups, where possible:

- Pregnancy self-test point of access (eg, pharmacy, online, CHW distribution).

- Type of pregnancy test.

- Population (eg, age, marital status, rural/urban).

- Vulnerabilities (eg, obesity, socioeconomic status, poverty, disability, literacy/educational level).

- Gestational age.

- High-income versus low or middle-income countries. Data were summarised in GRADE Evidence Profile tables using GRADEPro, where RCT data were available for a given outcome, we prioritised that over observational data.

\section{Values and preferences review}

The same search terms and screening process were used to identify studies to be included in the values and preferences review. Studies were included in this review if they presented primary data examining preferences of individuals regarding urine self-testing for pregnancy. We focused on studies examining the values and preferences of women and adolescent girls who were self-testing for pregnancy or individuals who were potential candidates for such self-testing, but we also included studies examining the values and preferences of health workers and other stakeholders. In this section, we also considered issues related to ability to access (by age, gender or other factors). These could include legal restrictions around who can access, for example, by age, requirement to be married to purchase, etc. These could also include practical barriers, for example, is it harder for adolescents to access self-tests because they are not allowed out on their own, they have no money, etc), informed decision-making, coercion, confidentiality, self-determination, health decision-making to terminate or maintain pregnancy (and discussion with partner if appropriate) and seeking redress. These studies could be qualitative or quantitative in nature, but had to present primary data collectionthink pieces and review articles were not included. Values and preferences literature was summarised qualitatively 
and organised by study design and methodology, location and population.

\section{Cost review}

The same search terms and screening process were used to identify studies to be included in the cost review. Studies would have been included in this review if they presented primary data comparing costing, cost-effectiveness, costutility or cost-benefit of the intervention and comparison listed in the PICOs above or if they presented costeffectiveness of the intervention as it relates to the PICO outcomes listed above. If cost literature had been found, we would have summarised it qualitatively. We planned to classify cost literature into four categories (health sector costs, other sector costs, patient/family costs and productivity impacts) and within each category would have organised by study design/methodology, location and population.

\section{Patient and public involvement}

Several of the authors are current or previous users of pregnancy self-tests. Feedback was also received from the WHO patient safety working group. Patients were involved in a global survey of values and preferences conducted to inform the WHO guideline on self-care interventions; they, thus play a significant role in the overall recommendation informed by this review.

\section{RESULTS}

Our search yielded 414 unique references, of which 62 were retained for full-text review (figure 1). Ultimately, we identified six that met the inclusion criteria for the effectiveness review, 18 values and preferences studies and no cost studies.

\section{Effectiveness review}

Overall, six studies met the inclusion criteria for the effectiveness review. This included five RCTs $^{5}{ }^{18-21}$ and one observational study. ${ }^{22}$ Table 1 presents descriptive data from the five RCTs. Four of the RCTs, conducted in a diverse range of countries (India, Vietnam, Austria, Finland, Norway, Sweden, Moldova and Uzbekistan), were conducted among 5493 individuals receiving medical abortion. ${ }^{18-21}$ These RCTs randomised clients to abortion follow-up with home pregnancy testing and a phone call, versus abortion follow-up with the traditional clinic visit, usually with ultrasound confirmation of successful termination. The fifth RCT, conducted in Madagascar, randomised CHWs to receive pregnancy tests to use with their clients versus the standard of care, which the authors said had historically been pregnancy testing available only at clinics. ${ }^{5}$

The RCTs provided data for two of our PICO outcomes: (1) appropriate clinical follow-up and (2) gestational age at pregnancy awareness (knowledge of pregnancy) and at presentation for antenatal care or abortion. The nonrandomised observational study provided data only for

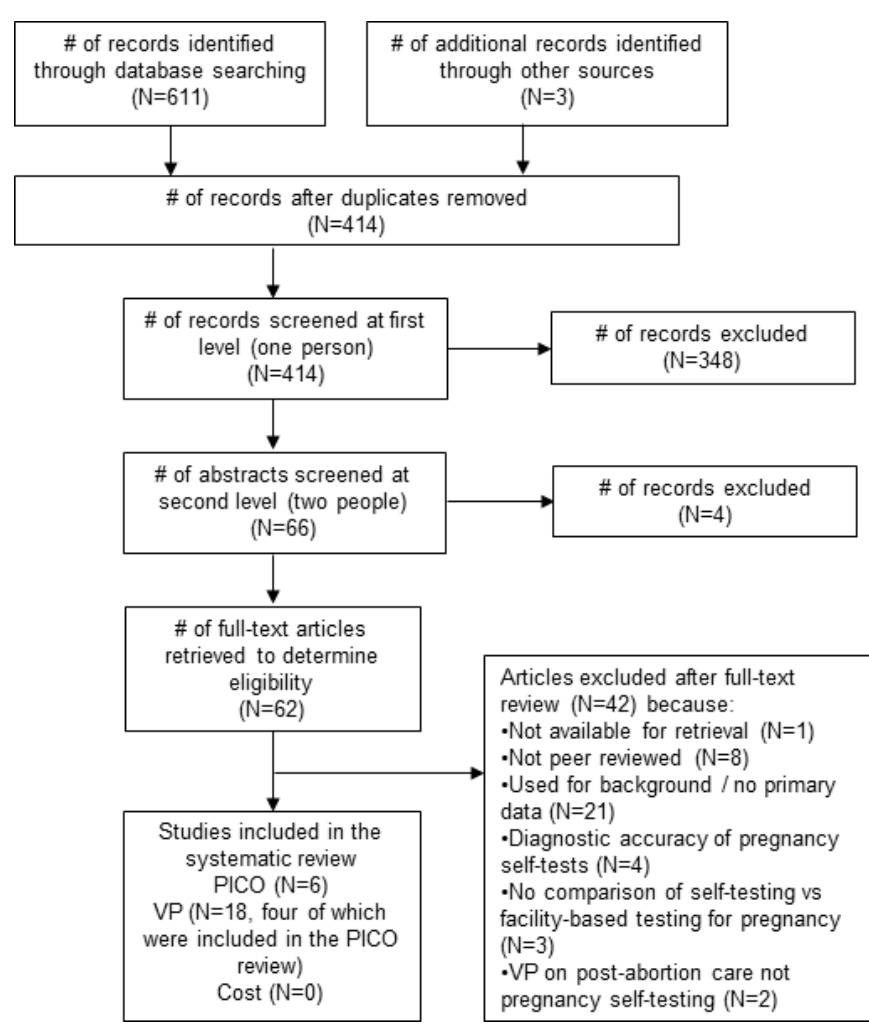

Figure 1 PRISMA flowchart showing disposition of citations through the search and screening process. PICO, populationintervention-comparison-outcome; PRISMA, Preferred Reporting Items for Systematic Reviews and Meta-Analyses; VP, values and preferences.

the same outcome under appropriate clinical follow-up, so per our protocol, we present only the RCT data here.

Appropriate clinical follow-up was assessed in the four postabortion RCTs as loss to follow-up, meaning the client did not return for their follow-up visit or was not able to be contacted by phone. One RCT from Vietnam found dramatically improved follow-up in the pregnancy self-testing arm, with only $0.6 \%$ of individuals lost compared with $8.1 \%$ lost in the comparison arm. ${ }^{19}$ The other three RCTs found no statistically significant differences between study arms. ${ }^{18} 2021$ In India, a study of 731 postabortion participants from urban and rural Rajasthan found loss to follow-up was $3.7 \%$ in the self-testing arm compared with $4.8 \%$ in the clinic arm. ${ }^{18}$ In urban Austria, Finland, Norway and Sweden, loss to follow-up among 929 postabortion participants was $19.5 \%$ in the self-testing arm compared with $22.1 \%$ in the clinic arm. ${ }^{20}$ Finally, the largest study of 2400 postabortion participants from Moldova and Uzbekistan found $<1 \%$ in the self-testing arm compared with no loss to follow-up in the clinic arm. $^{21}$

When the four studies were combined in meta-analysis, there was no significant difference between study arms in loss to follow-up (pooled risk ratio: $0.479,95 \%$ CI 0.155 to 1.480) (figure 2). Heterogeneity was substantial, with an $\mathrm{I}^{2}$ of 87 . Stratification by high-income versus low-income and middle-income settings did not yield meaningful 
Table 1 Description of RCTs included in the effectiveness review

\begin{tabular}{|c|c|c|c|c|c|}
\hline Study & Location & $\begin{array}{l}\text { Sample } \\
\text { size }\end{array}$ & Intervention & Comparator & Outcomes \\
\hline \multicolumn{6}{|c|}{ Women who had a medical abortion } \\
\hline Ngoc et al ${ }^{19}$ & $\begin{array}{l}\text { Vietnam (Ho Chi Minh } \\
\text { City and Hanoi) }\end{array}$ & $N=1433$ & \multirow{2}{*}{$\begin{array}{l}\text { Abortion follow- } \\
\text { up with home } \\
\text { pregnancy testing } \\
\text { and phone call }\end{array}$} & \multirow{2}{*}{$\begin{array}{l}\text { Abortion } \\
\text { follow-up with } \\
\text { clinic visit }\end{array}$} & \multirow{2}{*}{$\begin{array}{l}\text { 2. Appropriate clinical follow- } \\
\text { up }\end{array}$} \\
\hline Platais et $a l^{21}$ & $\begin{array}{l}\text { Moldova (Chisinau, } \\
\text { Balti, and Drochia), } \\
\text { Uzbekistan (Tashkent) }\end{array}$ & $\mathrm{N}=2400$ & & & \\
\hline
\end{tabular}

CHWs, community health workers; RCTs, randomised controlled trials.

differences; no further planned stratifications were possible, given the small number of similar studies identified for this outcome.

Appropriate clinical follow-up was assessed in the CHW RCT as the mean number of clients at risk of pregnancy who received antenatal counselling at their CHW visit per CHW. This was higher in the intervention group (mean difference: 0.39 clients more, $95 \%$ CI 0.14 more to 0.64 more).

Gestational age at pregnancy awareness (knowledge of pregnancy) and at presentation for antenatal care or abortion was also measured in the CHW RCT as the mean number of clients at risk of pregnancy who knew they were pregnant by the end of the visit per CHW. This was higher in the intervention group (mean difference: 0.86 clients more, $95 \%$ CI 0.59 more to 1.13 more).

Table 2 presents the GRADE evidence profile for the RCT outcomes. The four postabortion RCTs were rated down for indirectness of the population, as we were

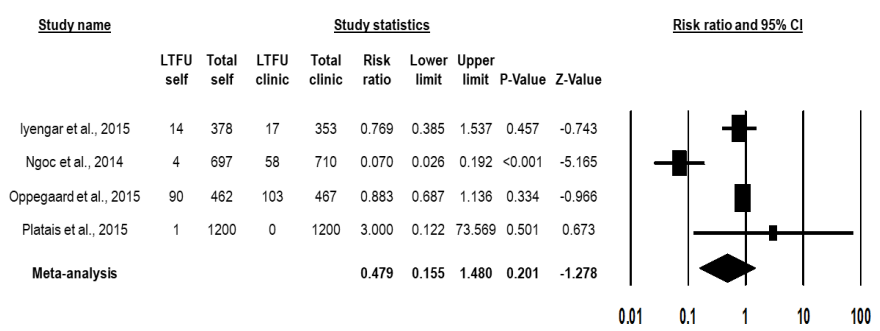

Figure 2 Meta-analysis of loss to follow-up (LTFU) among clients following medical abortion, comparing those receiving follow-up at home vs a return clinic visit. interested in all users of pregnancy self-tests while these studies focused on a specific subset of users following abortion. They were also rated down for inconsistency, as meta-analytic results across the four studies showed both the possibility of appreciable benefit and appreciable harm. This resulted in low certainty evidence. The CHW study was downgraded for risk of bias due to self-reporting of outcomes that may have been affected by self-report.

For our outcome of missed ongoing pregnancy, several studies of medical abortion follow-up looked at incomplete abortion. However, we did not consider this outcome measure as 'missed' pregnancies, as all were identified through the study protocols, except possibly for those lost to follow-up.

No studies measured our other outcomes of interest: self-efficacy, self-determination, autonomy and empowerment; mental health and well-being; adverse events and social harms; and device-related issues.

\section{Values and preferences review}

Overall, 18 studies were included in the values and preferences review. ${ }^{718-2123-35}$ Table 3 provides descriptive data and key findings of these studies.

There were 12 quantitative studies (all cross-sectional surveys) and four qualitative studies. For populations, six studies included general pregnancy test users or volunteers, while 12 studies followed individuals after they received a medical abortion with at-home follow-up including a home pregnancy test. No studies were identified with health workers or other stakeholders. 


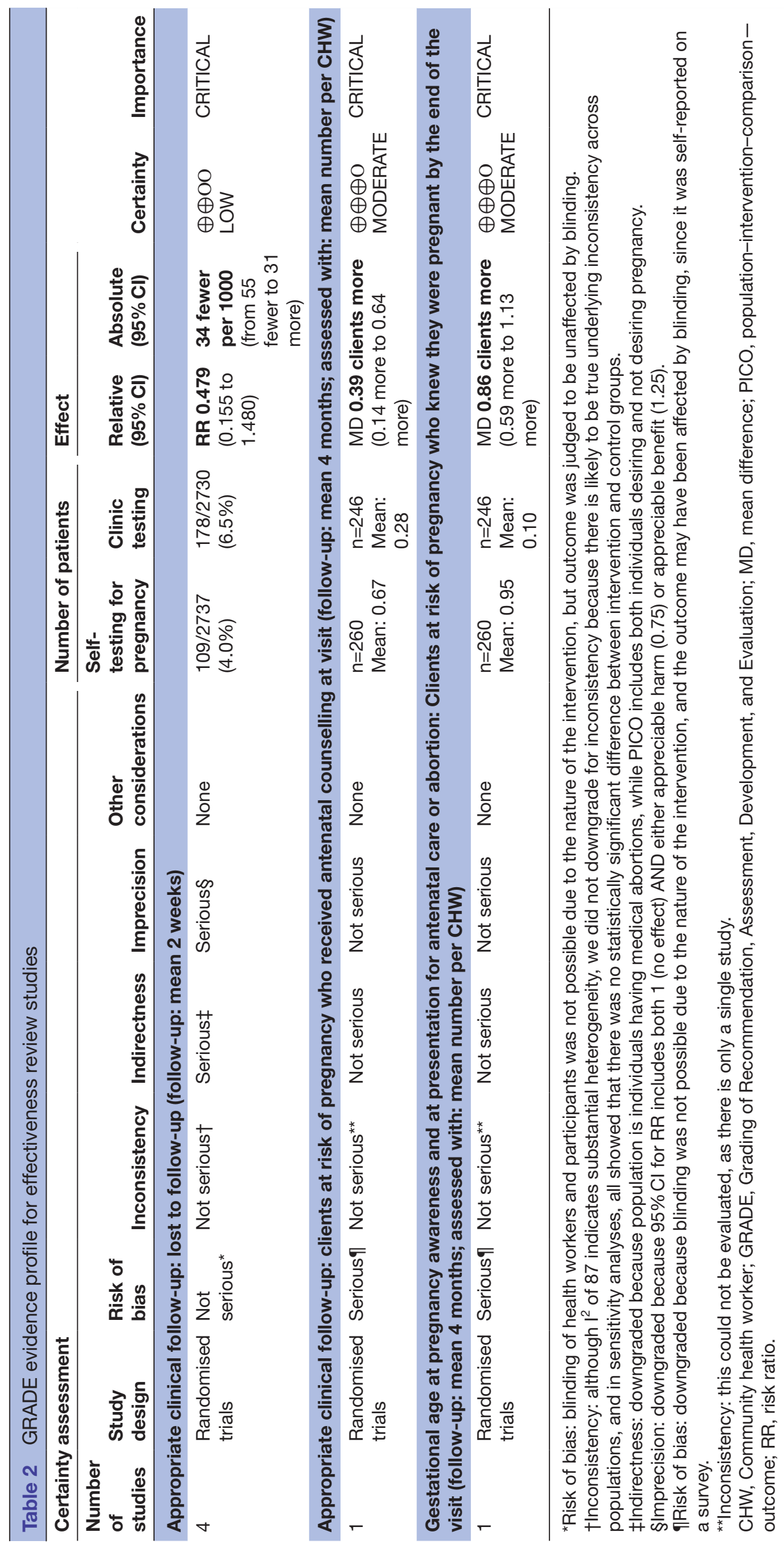




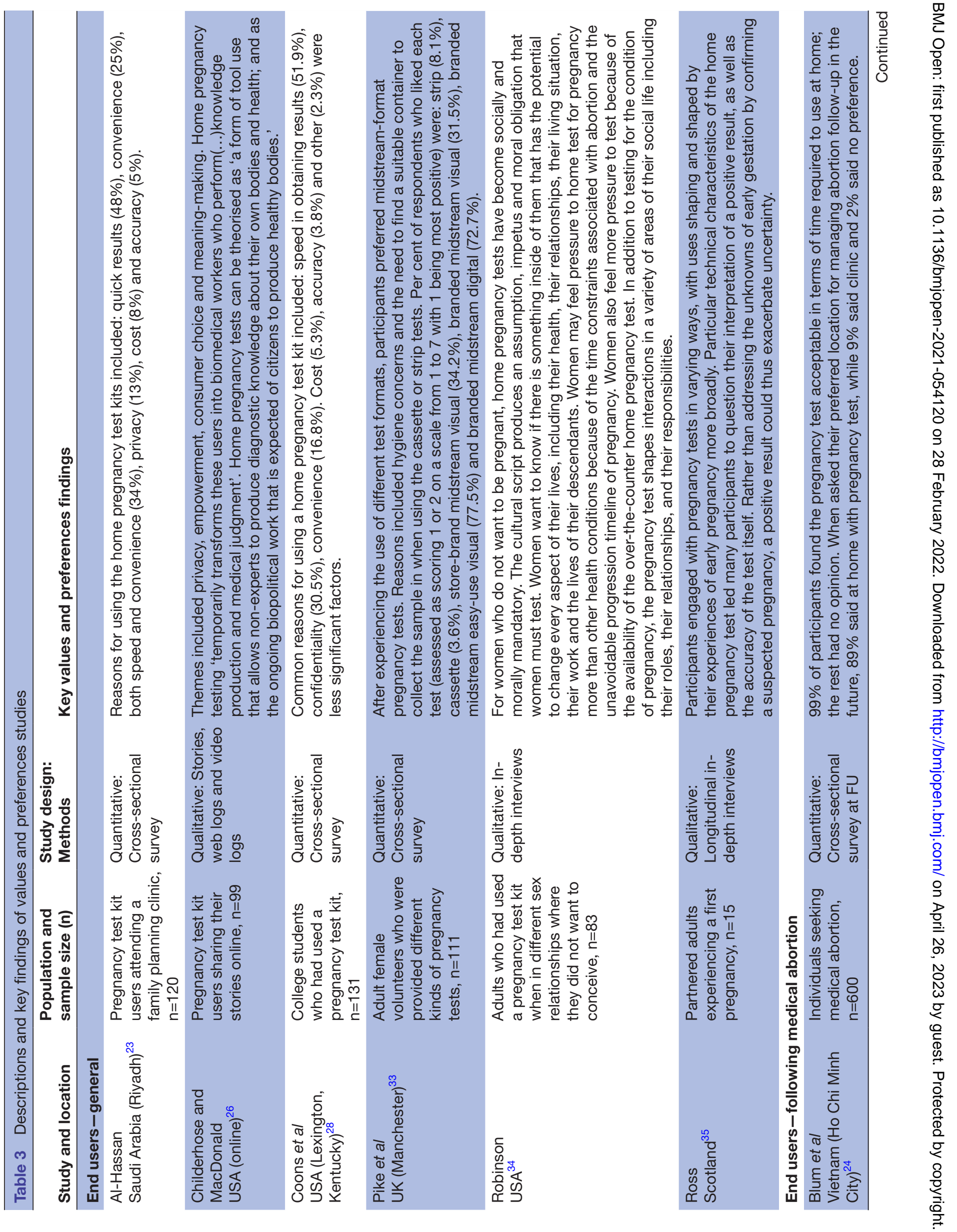




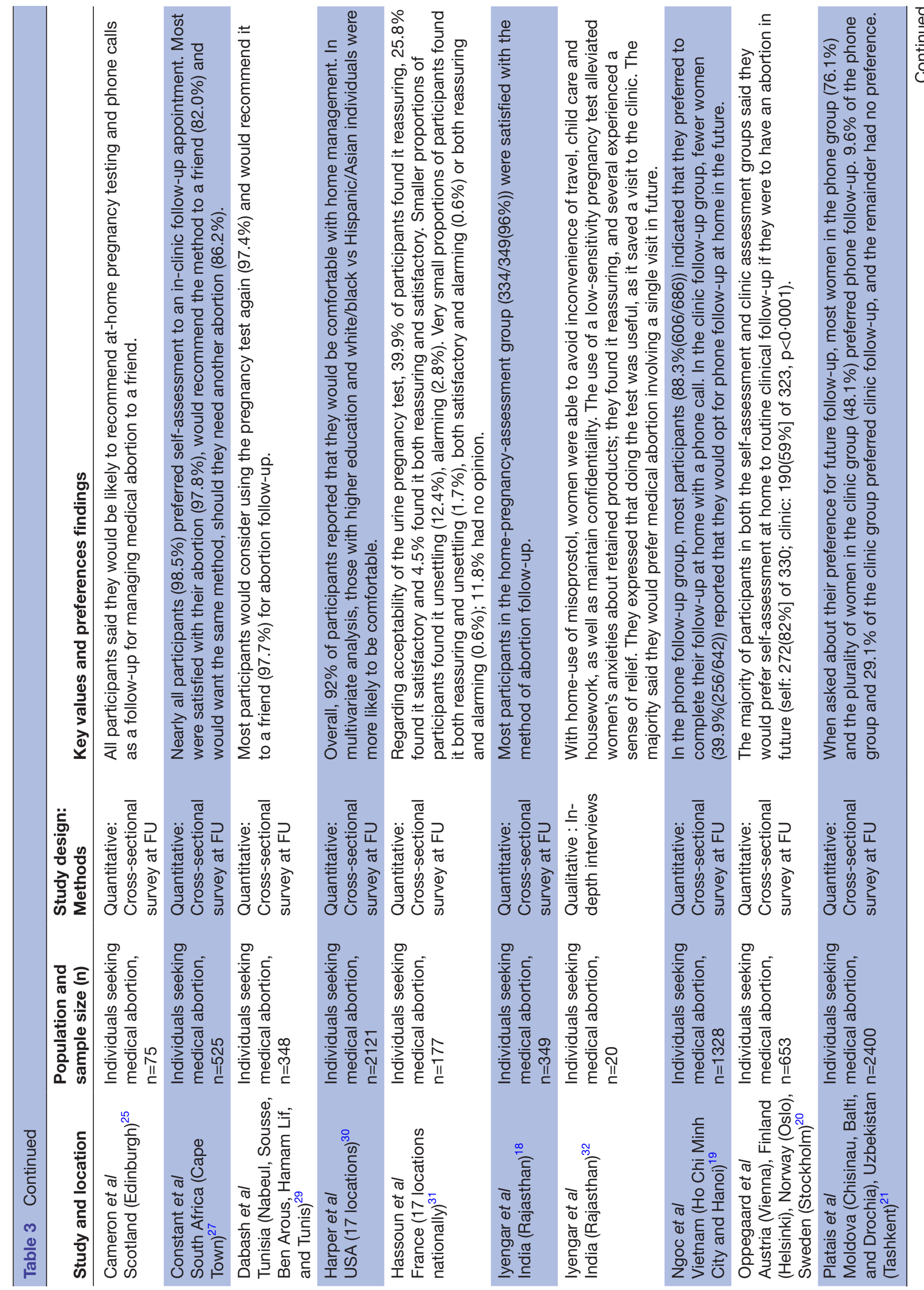


The studies were conducted in diverse locations: USA $(n=5)$, UK $(n=3)$, India $(n=2)$, Vietnam $(n=2)$ and one each in Austria, Finland, France, Moldova, Norway, Saudi Arabia, South Africa, Sweden, Tunisia and Uzbekistan.

Among general pregnancy test users, most studies found support for pregnancy tests. Reasons why individuals liked pregnancy tests included getting quick results, convenience, confidentiality/privacy, cost and accuracy. One study found a preference for midstream result tests when individuals were asked to try a range of tests. ${ }^{27}$ This same study found that certain types of tests were less preferred due to perceived cleanliness and convenience.

Among individuals having medical abortions, across all studies, most individuals receiving home management with a pregnancy test said they would prefer this option in the future. This ranged from $76.1 \%$ (Moldova and Uzbekistan) to $98.5 \%$ (South Africa). In two trials with clinic comparison groups, home management was still the most preferred option among participants in the clinic arms. When asked, clear majorities of participants across studies said they found home management acceptable and would recommend it to a friend.

While most values and preferences studies focused on acceptability, ease of use and reasons for preferring pregnancy self-testing over other options, few studies also presented findings on our other areas of interest: ability to access, informed decision-making, coercion, confidentiality, self-determination, health decision-making and seeking redress. One study from the USA found themes of privacy, empowerment, consumer choice and meaning-making among women using pregnancy tests. ${ }^{22}$ Other qualitative studies from the USA and Scotland noted that pregnancy tests shape individuals' relationships with their bodies and their social roles, relationships and responsibilities; ${ }^{28}{ }^{29}$ one theorised that testing is seen as socially and morally mandatory as 'the cultural script produces an assumption, impetus and moral obligation that women must test' to know what is inside their bodies and how it may shape their lives. ${ }^{28}$ Finally, one study from India found that managing abortion at home meant that women were able to avoid the inconvenience of travel, childcare and housework as well as maintain confidentiality. ${ }^{26}$ The pregnancy test alleviated women's anxieties: they found it reassuring, and several experienced a sense of relief.

\section{Cost review}

No studies presented primary data examining costeffectiveness, cost-utility or cost-benefit for pregnancy self-testing.

\section{DISCUSSION}

Pregnancy self-testing has become common practice in many high-income and middle-income settings, though is still not widely available in many low-income settings globally. Increased access and availability of pregnancy self-tests for home use can help confirm a pregnancy and 
engage with the health system for improved reproductive, maternal and newborn health outcomes. Pregnancy self-tests are also an important self-care intervention that increase agency and autonomy in sexual and reproductive decision-making. This systematic review is the first to our knowledge to summarise the available literature on effectiveness, values and preferences and cost for this important intervention.

In the effectiveness review, we found low-certainty evidence from four RCTs following individuals after medical abortion showing no difference in loss to follow-up with self-testing for pregnancy at home compared with a return clinic visit, although individual study results varied, and one did show substantial improvements in loss to follow-up with pregnancy self-testing. Moderate-quality evidence from one RCT showed that when CHWs were given pregnancy tests to provide to clients at home, there was a significant increase in the mean number of clients per CHW at risk of pregnancy who knew they were pregnant by the end of the visit and who received antenatal counselling. There was no data on missed ongoing pregnancy; self-efficacy, self-determination, autonomy and empowerment; mental health and well-being; adverse events and social harms or device-related issues. Given the ubiquity of self-testing for pregnancy in many settings, it is perhaps unsurprising that this intervention has not been studied widely in a comparative way except in specific circumstances. Given the positive findings in these two settings (postabortion care and CHW programmes), there is evidence that wider use of home pregnancy tests could have beneficial outcomes within health systems. However, fact that almost all data came from postabortion care setting limits the generalisability of our findings and the conclusions that can be drawn about public health benefits of this intervention. In addition, the certainty of evidence in the context of postabortion care was limited by the small number of studies combined in meta-analysis and high heterogeneity.

Furthermore, our effectiveness review only compared studies where individuals had access to home pregnancy tests versus facility-based testing. We excluded studies comparing groups of individuals given home pregnancy tests compared with those given nothing, or standard of care. Several trials have examined this latter question, asking whether having a home-pregnancy test on hand would cause women to have a lower threshold of suspicion for pregnancy, to test for pregnancy more often or to identify pregnancies earlier. For example, one RCT among low-income women in the USA found that participants given home pregnancy tests did suspect pregnancy more often and test for pregnancy more often than control participants. ${ }^{36}$ However, another larger RCT in the USA found no difference between arms in mean time at first pregnancy testing or mean gestational weeks at first positive pregnancy test. ${ }^{37}$ In both these cases, control participants presumably had at least some access to overthe-counter home pregnancy tests but results do speak to the potential impact of expanded access to such tests.
For values and preferences literature, 18 studies from diverse populations globally found that most individuals supported pregnancy self-testing. Reasons why individuals liked pregnancy tests included getting quick results, convenience, confidentiality/privacy, cost and accuracy. Mid-stream urine tests were preferred. Most individuals receiving postabortion home management with a pregnancy test said that they would prefer this option in the future. Although no data from health workers were identified, these positive findings from end users support broader access to pregnancy self-testing.

Our review identified no comparative cost data on pregnancy self-testing. Clearly, costs of pregnancy selftests will vary by setting and specific product but should generally fall within the range of other over-the-counter maternal health products. Considerations of cost should include not only the cost of the test for the end user but also the full range of health sector costs (eg, costs due to delayed pregnancy care), other sector costs and productivity impacts (eg, labour and workforce issues). Creative ways of expanding access to pregnancy self-testing within existing healthcare systems, such as using CHWs, would benefit from including cost and cost-effectiveness assessments.

This review had strengths and limitations. While we conducted a thorough and systematic search, we only included peer-reviewed literature; we, thus, may have missed grey literature. Strengths also included our double extraction of data and inclusion of not only effectiveness literature but also literature on values and preferences and costs. However, our findings across these three areas are limited by the literature available. We identified no cost data. Importantly, effectiveness data were heavily skewed towards literature on postabortion care, representing only a fraction of individuals using pregnancy self-tests globally. While our meta-analysis summarises the findings of the four effectiveness studies, it may provide a false sense of certainty that this represents the true effect. Furthermore, we only had sufficient studies to meta-analyse the outcome 'lost to follow-up' after abortion, which provides just one specific way of assessing the overall outcome category of 'appropriate clinical follow-up'. Further research on the effectiveness of pregnancy self-testing (especially on outcomes for which we found no comparative data, that is, missed ongoing pregnancy; self-efficacy, self-determination, autonomy and empowerment; mental health and well-being; adverse events and social harms and device-related issues), values and preferences and costs is needed, particularly in lowresource settings and in contexts where people would benefit from greater accessibility.

Ultimately, the WHO Consolidated guideline on selfcare interventions for health and well-being published in 2021 included the following recommendation: 'WHO recommends making self-testing for pregnancy available as an additional option to health worker-led testing for pregnancy, for individuals seeking pregnancy testing (strong recommendation; very low certainty evidence) ${ }^{38}$ 


\section{CONCLUSION}

Pregnancy self-testing is acceptable and valued by end users. Ensuring universal access to pregnancy self-testing may encourage more women and girls to seek early antenatal care, which is a critical opportunity for health workers to deliver care and support during pregnancy, thus contributing to better health outcomes for women, newborn and children. Novel ways of delivering pregnancy self-tests, including through CHW programmes, show promise on improving sexual and reproductive health and rights outcomes and should be considered for further study and expansion. In the current context of the COVID-19 pandemic, greater availability of pregnancy self-testing can also help maintain the continuity and quality of sexual and reproductive health services.

Acknowledgements We thank Johns Hopkins graduate students Hunied Kautsar, Cynthia Li, and Praise Olatunde for their help screening citations and coding articles for this review and Xuhao Yang for copy-editing. We also thank Laura Ferguson, Nandi Siegfried, Leopold Ouedraogo and Hayfa Elamin for their thoughtful comments on the protocol or draft manuscript. We gratefully acknowledge financial support of The Children's Investment Fund Foundation (CIFF).

Contributors MN conceptualised the study following discussion with $\mathrm{KG}$ and other WHO Regional Advisors from the European and African Regions. CEK and PTY designed the protocol, with feedback from MN. PTY ran the database search and oversaw the search, screening, full text review, and data extraction process. CEK and PTY drafted the manuscript. All authors reviewed the draft, provided critical review, and read and approved the final manuscript. The corresponding author, as guarantor, accepts full responsibility for the finished article has access to any data and controlled the decision to publish. The corresponding author attests that all listed authors meet the authorship criteria and that no others meeting the criteria have been omitted.

Funding This review was commissioned by the World Health Organization and funded by The Children's Investment Fund Foundation (CIFF).

\section{Competing interests None declared.}

Patient and public involvement Patients and/or the public were involved in the design, or conduct, or reporting, or dissemination plans of this research. Refer to the Methods section for further details.

Patient consent for publication Not applicable.

Ethics approval This study does not involve human participants. Ethical approval was not required for this systematic review, since all data came from published articles.

Provenance and peer review Not commissioned; externally peer reviewed.

Data availability statement All data relevant to the study are included in the article or uploaded as supplementary information. Extracted data are available on request to the corresponding author.

Supplemental material This content has been supplied by the author(s). It has not been vetted by BMJ Publishing Group Limited (BMJ) and may not have been peer-reviewed. Any opinions or recommendations discussed are solely those of the author(s) and are not endorsed by BMJ. BMJ disclaims all liability and responsibility arising from any reliance placed on the content. Where the content includes any translated material, BMJ does not warrant the accuracy and reliability of the translations (including but not limited to local regulations, clinical guidelines, terminology, drug names and drug dosages), and is not responsible for any error and/or omissions arising from translation and adaptation or otherwise.

Open access This is an open access article distributed in accordance with the Creative Commons Attribution Non Commercial (CC BY-NC 4.0) license, which permits others to distribute, remix, adapt, build upon this work non-commercially, and license their derivative works on different terms, provided the original work is properly cited, appropriate credit is given, any changes made indicated, and the use is non-commercial. See: http://creativecommons.org/licenses/by-nc/4.0/.

\section{ORCID iD}

Ping Teresa Yeh http://orcid.org/0000-0002-7425-0382
REFERENCES

1 Ehrenkranz JRL. Home and point-of-care pregnancy tests: a review of the technology. Epidemiology 2002;13 Suppl 3:S15-18.

2 Gronowski AM, Cervinski M, Stenman U-H, et al. False-Negative results in point-of-care qualitative human chorionic gonadotropin (hCG) devices due to excess hCGbeta core fragment. Clin Chem 2009;55:1389-94.

3 Nerenz RD, Song H, Gronowski AM. Screening method to evaluate point-of-care human chorionic gonadotropin (hCG) devices for susceptibility to the hook effect by hCG $\beta$ core fragment: evaluation of 11 devices. Clin Chem 2014;60:667-74.

4 Bastian LA, Nanda K, Hasselblad V, et al. Diagnostic efficiency of home pregnancy test kits. A meta-analysis. Arch Fam Med 1998;7:465-9.

5 Comfort AB, Juras RC, Bradley SEK, et al. Do home pregnancy tests bring women to community health workers for antenatal care counselling? A randomized controlled trial in Madagascar. Health Policy Plan 2019;34:566-73.

6 Comfort AB, Chankova S, Juras R, et al. Providing free pregnancy test kits to community health workers increases distribution of contraceptives: results from an impact evaluation in Madagascar. Contraception 2016:93:44-51.

7 Shochet T, Comstock IA, Ngoc NTN, et al. Results of a pilot study in the U.S. and Vietnam to assess the utility and acceptability of a multi-level pregnancy test (MLPT) for home monitoring of hCG trends after assisted reproduction. BMC Womens Health 2017;17:67.

8 Baiju N, Acharya G, D'Antonio F, et al. Effectiveness, safety and acceptability of self-assessment of the outcome of first-trimester medical abortion: a systematic review and meta-analysis. BJOG 2019;126:1536-44.

9 Schmidt-Hansen M, Cameron S, Lohr PA, et al. Follow-up strategies to confirm the success of medical abortion of pregnancies up to 10 weeks' gestation: a systematic review with meta-analyses. Am J Obstet Gynecol 2020;222:551-63.

10 World Health Organization. Regional meeting for engaging countries and strengthening partnerships towards better maternal and child health, Amman, Jordan. Cairo, Egypt: WHO Regional Office for the Eastern Mediterranean, 2018. http://www.emro.who.int/reproductivehealth-network/rhrn-events/partnerships-maternal-child-health.html

11 World Health Organization. Who consolidated guideline on selfcare interventions for health: sexual and reproductive health and rights. Geneva, Switzerland: WHO, 2019. https://www.who.int/ reproductivehealth/publications/self-care-interventions/en/

12 Moher D, Liberati A, Tetzlaff J, et al. Preferred reporting items for systematic reviews and meta-analyses: the PRISMA statement. PLoS Med 2009;6:e1000097.

13 World Health Organization. Who Handbook for Guideline development. 2nd ed. Geneva, Switzerland: WHO, 2014. https:// apps.who.int/iris/handle/10665/145714

14 GRADE Working Group. Grade Handbook. Ontario, Canada: McMaster University and Evidence Prime, Inc, 2013. https://gdt. gradepro.org/app/handbook/handbook.html

15 Higgins JPT, Thomas J, Chandler J. Cochrane Handbook for systematic reviews of interventions. version 6.0, 2019. Available: www.training.cochrane.org/handbook

16 Kennedy CE, Fonner VA, Armstrong KA, et al. The evidence project risk of bias tool: assessing study rigor for both randomized and nonrandomized intervention studies. Syst Rev 2019;8:3.

17 Borenstein M, Hedges L, Higgins J. Comprehensive meta-analysis version 3. Englewood, NJ: Biostat, 2013.

18 lyengar K, Paul M, lyengar SD, et al. Self-Assessment of the outcome of early medical abortion versus clinic follow-up in India: a randomised, controlled, non-inferiority trial. Lancet Glob Health 2015;3:e537-45.

19 Ngoc NTN, Bracken H, Blum J, et al. Acceptability and feasibility of phone follow-up after early medical abortion in Vietnam: a randomized controlled trial. Obstet Gynecol 2014;123:88-95.

20 Oppegaard KS, Qvigstad E, Fiala C, et al. Clinical follow-up compared with self-assessment of outcome after medical abortion: a multicentre, non-inferiority, randomised, controlled trial. Lancet 2015;385:698-704.

21 Platais I, Tsereteli T, Comendant R, et al. Acceptability and feasibility of phone follow-up with a semiquantitative urine pregnancy test after medical abortion in Moldova and Uzbekistan. Contraception 2015;91:178-83.

22 Chen MJ, Rounds KM, Creinin MD, et al. Comparing office and telephone follow-up after medical abortion. Contraception 2016;94:122-6.

$23 \mathrm{Al}-\mathrm{Hassan}$ MI. Qualitative determination of pregnancy by home pregnancy test kit: a survey on purchase and use of kits in Saudi community. Res J Med Sci 2011;5:224-7. 
24 Blum J, Sheldon WR, Ngoc NTN, et al. Randomized trial assessing home use of two pregnancy tests for determining early medical abortion outcomes at 3,7 and 14days after mifepristone. Contraception 2016;94:115-21.

25 Cameron ST, Glasier A, Dewart $\mathrm{H}$, et al. Telephone follow-up and self-performed urine pregnancy testing after early medical abortion: a service evaluation. Contraception 2012;86:67-73.

26 Childerhose JE, Macdonald ME. Health consumption as work: the home pregnancy test as a domesticated health tool. Soc Sci Med 2013;86:1-8.

27 Constant D, Harries J, Daskilewicz K, et al. Is self-assessment of medical abortion using a low-sensitivity pregnancy test combined with a checklist and phone text messages feasible in South African primary healthcare settings? A randomized trial. PLoS One 2017; 12:e0179600.

28 Coons SJ, Churchill L, Brinkman ML. The use of pregnancy test kits by college students. J Am Coll Health 1990;38:171-5.

29 Dabash R, Shochet T, Hajri S, et al. Self-Administered multi-level pregnancy tests in simplified follow-up of medical abortion in Tunisia. BMC Womens Health 2016;16:49.

30 Harper C, Ellertson C, Winikoff B. Could American women use mifepristone-misoprostol pills safely with less medical supervision? Contraception 2002;65:133-42.
31 Hassoun D, Périn I, Hiên $\mathrm{H}$, et al. Feasibility of self-performed urine pregnancy testing for follow-up after medical abortion. Eur J Obstet Gynecol Reprod Biol 2016;197:174-8.

32 lyengar K, Klingberg Allvin M, lyengar SD, et al. "Who Wants to Go Repeatedly to the Hospital?" Perceptions and Experiences of Simplified Medical Abortion in Rajasthan, India. Glob Qual Nurs Res 2016;3:233339361668307.

33 Pike J, Godbert S, Johnson S. Comparison of volunteers' experience of using, and accuracy of reading, different types of home pregnancy test formats. Expert Opin Med Diagn 2013;7:435-41.

34 Robinson $\mathrm{JH}$. What the pregnancy test is testing. $\mathrm{Br} \mathrm{J}$ Sociol 2020;71:460-73.

35 Ross E. Provisionally pregnant: uncertainty and interpretive work in accounts of home pregnancy testing. Health 2018;22:87-105.

36 Nettleman MD, Ayoola AB, Brewer JR. Utilization of home pregnancy testing among women at risk for unintended pregnancy. Womens Health Issues 2009;19:263-7.

37 Wise LA, Wang TR, Willis SK, et al. Effect of a home pregnancy test intervention on cohort retention and pregnancy detection: a randomized trial. Am J Epidemiol 2020;189:773-8.

38 World Health Organization. Who consolidated guideline on self-care interventions for health and wellbeing. Geneva, Switzerland: WHO, 2021. https://app.magicapp.org/\#/guideline/Lr21gL 